\title{
A Presentation for the Virasoro and Super-Virasoro Algebras
}

D. B. Fairlie ${ }^{1}$, J. Nuyts ${ }^{2}$, and C. K. Zachos ${ }^{3 \star}$

${ }^{1}$ Department of Mathematical Sciences, University of Durham, Durham DH1 3LE, England

${ }^{2}$ Faculté des Sciences, Université de l'État à Mons, 19 Avenue Maistriau, Mons, Belgium 7000

${ }^{3}$ High Energy Physics Division, Argonne National Laboratory, Argonne, IL 60439, USA

\begin{abstract}
It is shown that the entire Virasoro, Ramond and Neveu-Schwarz algebras can each be constructed from a finite number of well-chosen generators satisfying a small number of conditions. The most economical sets consist of just two starting generators in all cases, subject to eight conditions for the Virasoro case, five conditions for the Ramond case, and nine conditions for the Neveu-Schwarz case.
\end{abstract}

\section{Introduction}

The Virasoro algebra [1,2], for example

$$
\left[L_{m}, L_{n}\right]=(m-n) L_{m+n}+c\left(m^{3}-m\right) \delta_{m+n, 0},
$$

(where the indices $m$ and $n$ run over the positive and negative integers) is normally contrasted to the classical algebras, as their closure contains only a finite number of generators. In one respect, however, the Virasoro algebra resembles these algebras, even down to their simple prototype $S U(2)$ : a small number of generators, in this case 2 , suffices to define the rest through appropriate chains of commutations. The entire algebra then follows inductively by use of the Jacobi identities, provided that a finite number of independent commutation relations be imposed in each case. Alternatively, the structure of the entire algebra is determined as a solution of these commutation conditions, regarded as equations for the starting generators. For $S U(2)$, this is evident; given the two starting generators $T_{1}$ and $T_{2}$, the third generator $T_{3}$ is defined by

$$
\text { (Def.) } T_{3}=-i\left[T_{1}, T_{2}\right]
$$

\footnotetext{
* Work supported by the U.S. Department of Energy, Division of High Energy Physics, Contract W-31109-ENG-38
} 
and the two conditions which specify the algebra are:

$$
\begin{array}{ll}
\text { (Cond. } i) & T_{1}=\left[T_{2},\left[T_{2}, T_{1}\right]\right], \\
\text { (Cond. } i \text { ) } & T_{2}=\left[T_{1},\left[T_{1}, T_{2}\right]\right] .
\end{array}
$$

The solutions to these equations yield all representations of $S U(2)$ and the constructions based on them.

The purpose of this paper is to prove that, starting for example from $L_{3}$ and $L_{-2}$ in the Virasoro case, the entire algebra (1) can be specified, provided that eight commutation conditions be posited. Our discussion below will first focus on defining all $L_{n}$ 's recursively out of two such, and then demonstrating how all remaining relations (1) are consequences of merely a set of 8 chosen from them. We shall defer discussion of alternative starting sets, general systematics, and significance to the last section of the paper, beginning with the above particular choice of starting generators for illustration purposes.

Using the same type of arguments, we shall then treat the Ramond and NeveuSchwarz [3] cases where commutation and anticommutation relations have to be used: fermion generators $F$ are introduced, obeying

$$
\begin{aligned}
& {\left[L_{n}, F_{a}\right]=(n / 2-a) F_{n+a},} \\
& \left\{F_{a}, F_{b}\right\}=2 L_{a+b}+c\left(4 a^{2}-1\right) \delta_{a+b, 0},
\end{aligned}
$$

where the new indices $a$ and $b$ run over the integers for the Ramond case and the half-integers for the Neveu-Schwarz case. We shall conclude that the Ramond algebra amounts to merely five conditions among two starting generators, and the Neveu-Schwarz algebra to nine conditions among two starting generators.

\section{The Virasoro Algebra}

Let us start with the most economical set of defining generators which allow the construction of the Virasoro algebra, i.e. just the two generators $L_{3}$ and $L_{-2}$.

Lemma 1. $L_{3}$ and $L_{-2}$ reach all the $L_{n}$ 's through iterative definitions of the type (2).

This is seen directly, by constructing

$$
\begin{aligned}
\text { (Def. 1) } & L_{1}=\frac{1}{5}\left[L_{3}, L_{-2}\right], \\
\text { (Def. 2) } & L_{-1}=\frac{1}{3}\left[L_{1}, L_{-2}\right], \\
\text { (Def. 3) } & L_{2}=\frac{1}{4}\left[L_{3}, L_{-1}\right], \\
\text { (Def. 4) } & L_{0}=\frac{1}{2}\left[L_{1}, L_{-1}\right],
\end{aligned}
$$

for the lowest values of the indices. The other operators are then defined recursively from there, as

$$
\text { (Def) } L_{n+1}=\frac{1}{(n-1)}\left[L_{n}, L_{1}\right] \text { for } n>2,
$$

for the positive indices larger than three; and

$$
\text { (Def) } L_{-n-1}=\frac{1}{(1-n)}\left[L_{-n}, L_{-1}\right] \text { for } n>1,
$$

for the negative indices smaller than minus two. 
A different starting set, and hence a different set of definitions, could have been used to define all $L_{n}$ 's, as will be discussed in the last section. Using the above definitions, our next task is to investigate which small subset of the commutation relations (1) guarantee the validity of all.

Our inductive tool will be the Jacobi identity $J(m, n, p)$,

$$
J(m, n, p): \quad\left[\left[L_{m}, L_{n}\right], L_{p}\right]+\left[\left[L_{n}, L_{p}\right], L_{m}\right]+\left[\left[L_{p}, L_{m}\right], L_{n}\right]=0 .
$$

A word of caution: at every step in the inductive process to follow, a given Jacobi identity can only be used if the three inner commutators, i.e. $\left[L_{m}, L_{n}\right] \ldots$ etc., are already known by a preceding step in the induction. The Jacobi identity then reduces to:

$$
J(m, n, p) \equiv(m-n)\left[L_{m+n}, L_{p}\right]+(n-p)\left[L_{n+p}, L_{m}\right]+(p-m)\left[L_{p+m}, L_{n}\right]=0,
$$

and will then be used in this form. As seen later, $m+n+p$ is the eigenvalue of each term in (8) under commutation with $L_{0}$. We call $m+n+p$ the level of this corresponding Jacobi identity.

We now point out a "parity" automorphism of the algebra which helps shorten the proofs to follow.

Lemma 2. The commutation relations (1) are invariant under the symmetry operation

$$
L_{n} \rightarrow-L_{-n}, \quad c \rightarrow-c
$$

All statements derived from sets possessing this symmetry will also be symmetric.

Lemma 3. The commutator of two operators of level $m$ and $n$ is of level $m+n$.

The proof follows directly from the Jacobi identity $J(0, m, n)$.

Lemma 4. The commutation relations of $L_{0}$ with all Virasoro operators follow from its commutation relations with $L_{3}$ and $L_{-2}$ :

$$
\begin{aligned}
\text { (Cond. 1) } \quad\left[L_{3}, L_{0}\right] & =3 L_{3}, \\
\text { (Cond. 2) } \quad\left[L_{-2}, L_{0}\right] & =-2 L_{-2} .
\end{aligned}
$$

This follows trivially from the definitions and from the previous lemma.

Lemma 5. Within the initially defined set (Def. 1-4) $L_{-2}, L_{-1}, L_{0}, L_{1}, L_{2}, L_{3}$, it is easy to see that two further conditions must be imposed:

$$
\begin{aligned}
\text { (Cond. 3) } \quad\left[L_{2}, L_{1}\right] & =L_{3}, \\
\text { (Cond. 4) } \quad\left[L_{2}, L_{-2}\right] & =4 L_{0}+6 c .
\end{aligned}
$$

Note that $\left[L_{2}, L_{-1}\right]=3 L_{1}$ follows from $J(1,-2,2)$, Conds. 3, 4, Defs. 1,2 , and the previous lemma. $c$ is defined as a mere number times the identity, so that it commutes with all the starting generators, and hence all operators. All the allowed Jacobi identities within this set are then satisfied, and the definition (6) extends to $n=2$.

It is sufficient to further impose the following 4 conditions $^{1}$ to completely

1 The special role of levels 5 and 7 has been noted in different contexts, such as string theory, by Y. Meurice in ref. [4] 
determine (1):

$\begin{aligned} \text { (Cond. 5) } & {\left[L_{3}, L_{2}\right] } & =L_{5} \\ \text { (Cond. 6) } & {\left[L_{5}, L_{2}\right] } & =3 L_{7} \\ \text { (Cond. 7) } & {\left[L_{-2}, L_{-3}\right] } & =L_{-5} \\ \text { (Cond. 8) } & {\left[L_{-2}, L_{-5}\right] } & =3 L_{-7} .\end{aligned}$

We shall prove (1) separately for several classes of relations, mindful of the algebra automorphism of Lemma 2, which holds for all commutators assumed or established so far, and hence will hold for all their consequences. The first class includes the commutators of operators with positive indices. Observe that, up to level $k \equiv m+n=4, m, n>0$, all commutators are known by the above.

Lemma 6. All the commutators of fixed total level $k \equiv m+n>4$, where $m, n>0$, can be obtained in terms of $L_{m+n}$ through their definitions, except for levels 5 and 7 where two new conditions (Cond. 5,6) have to be imposed.

Proof. This is shown by induction on $k$, based on the previous lemma. Assuming it holds for $k-1$, define the unknown commutators $X_{t}^{(k)}$ by

$$
X_{t}^{(k)} \equiv\left[L_{t+1}, L_{k-t-1}\right],
$$

where $t$ runs from $t=1$ to $t=r-2$, for $k=2 r$ even, and to $t=r-1$ for $k \equiv 2 r+1$ odd.

For $k \equiv 2 r$ even and greater than 4 , the $r-2$ Jacobi identities $J(1, s+1, k-s-2)$, $s=1, \ldots, r-2$, lead to a system of $r-2$ equations in the $r-2$ unknowns $X_{t}$, $t=1, \ldots, r-2$, of the form

$$
M_{s t}^{(k)} X_{t}^{(k)}=m_{s}^{(k)} L_{k}
$$

where

$$
M^{(k=2 r)}=\left(\begin{array}{ccccccc}
k-4 & 1 & 0 & 0 & \cdots & 0 & 0 \\
0 & k-5 & 2 & 0 & \cdots & 0 & 0 \\
0 & 0 & k-6 & 3 & \cdots & 0 & 0 \\
0 & 0 & 0 & k-7 & \cdots & 0 & 0 \\
\vdots & \vdots & \vdots & \vdots & \vdots & \vdots & \\
0 & 0 & 0 & 0 & \cdots & r & r-3 \\
0 & 0 & 0 & 0 & \cdots & 0 & r-1
\end{array}\right)
$$

and the vector $m_{s}^{(k=2 r)}$ has components

Since

$$
m_{s}^{(k=2 r)}=(2 s+3-k)(k-2) .
$$

$$
\operatorname{det} M^{(k=2 r)}=\frac{(k-4) !}{(r-2) !}
$$

is non-zero, (15) admits the known unique solution (1) for the $X_{t}^{(k)}$ 's in terms of $L_{k}$.

When $k$ is odd and larger than $3, k \equiv 2 r+1$, there are $r-1$ unknowns, but the Jacobi identities $J(1, s+1, k-s-2), s=1, \ldots, r-2$, provide only $r-2$ 
equations. The matrix in (16) now has to be supplemented by an extra row with the Jacobi identity $J(2,3, k-5)$, (and the last component of $m^{(k=2 r+1)}$ vanishes), so it becomes $(r-1) \times(r-1)$ :

$$
M^{(k=2 r+1)}=\left(\begin{array}{cccccccc}
k-4 & 1 & 0 & 0 & 0 & \cdots & 0 & 0 \\
0 & k-5 & 2 & 0 & 0 & \cdots & 0 & 0 \\
0 & 0 & k-6 & 3 & 0 & \cdots & 0 & 0 \\
0 & 0 & 0 & k-7 & 4 & \cdots & 0 & 0 \\
\vdots & \vdots & \vdots & \vdots & \vdots & \vdots & \vdots & \vdots \\
0 & 0 & 0 & 0 & 0 & \cdots & r-3 & 0 \\
0 & 0 & 0 & 0 & 0 & \cdots & r & r-2 \\
8-k & k-7 & 0 & 1 & 0 & \cdots & 0 & 0
\end{array}\right) .
$$

For $r=4$, the bottom row is $(-1,2,-1)$ instead. Again,

$$
\operatorname{det} M^{(k=2 r+1)}=(-1)^{r+1}(k-7)(k-2) k \frac{(r-2) !}{3 !} \neq 0
$$

for $r>3$. For $r=2,3$, i.e. $k=5,7$, the extra Jacobi identity does not exist to provide the additional row, and (Conds. 5,6) have to be imposed.

The same conclusions apply to the case $m, n<0$ by virtue of Lemma 2 and (Conds. 7,8). We proceed to study the commutation relations of negative with positive indices. First, the commutators of $L_{-1}$ with the $L_{m}(m>2)$ are obtained from the following lemma.

Lemma 7. The commutator of $L_{-1}$ with $L_{m}, m>2$ is obtained from the known commutators of $L_{-1}$ with $L_{1}$ and $L_{2}$, and of $L_{0}$ with $L_{m}$ by induction through the use of the Jacobi identity $J(-1,1, m)$, starting with $m=2$.

By the automorphism of Lemma 2, the commutators of $L_{1}$ with the negative $L_{-n}, n>2$ are likewise known. Thus the commutators of $L_{1}$ and $L_{-1}$ with all the $L_{n}$ 's are determined. The commutators of the negative and positive $L$ 's can finally be obtained inductively from the following lemma.

Lemma 8. The commutators of $L_{m}$ with $L_{-n}$ are obtainable in terms of $L_{m-n}$ for all $n, m>2$.

The $m=n>2$ and the $m=n+1>2$ commutators follow inductively by sequential use of $J(1, m,-m)$ and $J(-1, m+1,-m)$, starting with $m=2$. Now, given some $m$, assume the result holds for all positive $n$ smaller than or equal to this $m$; then, by virtue of $J(1, m,-n)$ and the above, the result follows inductively in $m$ (for all $n \leqq m$ ), starting with first value $m=2$ (induction basis). For $m<n$, the lemma follows by the above and the parity of Lemma 2 .

Finally, the lemmas are readily assembled to obtain:

Theorem 1. Given $L_{3}$ and $L_{-2}$ and the definitions of Eqs. (5), (6) and (7), with the eight conditions of Eqs. (11), (12) and (13), the entire Virasoro algebra (1) is satisfied. 


\section{The Ramond Algebra}

We now turn to a supersymmetric extension of (1), namely the Ramond algebra given by (4) when the indices $a, b$ run over the integers. In this algebra, since there are commutators and anticommutators, there are four types of graded Jacobi identities, involving respectively three $L$ 's, two $L$ 's and one $F$, one $L$ and two $F$ 's, and three $F$ 's. They are given by $(8)$ as well as $J 1(m, n, p), J 2(m, n, p)$, and $J 3(m, n, p)$, respectively; by convention, the $i$ in $J i(m, n, p)$ refers to the number of $F$ 's in the Jacobi identity and the $F$ indices are at the right.

$$
\begin{array}{ll}
J 1(m, n, p): & {\left[\left[L_{m}, L_{n}\right], F_{p}\right]+\left[\left[L_{n}, F_{p}\right], L_{m}\right]+\left[\left[F_{p}, L_{m}\right], L_{n}\right]=0,} \\
J 2(m, n, p): & \left\{\left[L_{m}, F_{n}\right], F_{p}\right\}+\left[\left\{F_{n}, F_{p}\right\}, L_{m}\right]-\left\{\left[F_{p}, L_{m}\right], F_{n}\right\}=0, \\
J 3(m, n, p): & {\left[\left\{F_{m}, F_{n}\right\}, F_{p}\right]+\left[\left\{F_{n}, F_{p}\right\}, F_{m}\right]+\left[\left\{F_{p}, F_{m}\right\}, F_{n}\right]=0 .}
\end{array}
$$

If all the inner commutators are known from a previous step in an induction, then these Jacobi identities reduce to the set (9) together with

$$
\begin{aligned}
& J 1(m, n, p) \equiv 2(m-n)\left[L_{m+n}, F_{p}\right]+(n-2 p)\left[F_{n+p}, L_{m}\right]+(2 p-m)\left[F_{p+m}, L_{n}\right]=0, \\
& J 2(m, n, p) \equiv 4\left[L_{m}, L_{n+p}\right]+(2 n-m)\left\{F_{m+n}, F_{p}\right\}+(2 p-m)\left\{F_{m+p}, F_{n}\right\}=0, \\
& J 3(m, n, p) \equiv\left[L_{m+n}, F_{p}\right]+\left[L_{n+p}, F_{m}\right]+\left[L_{p+m}, F_{n}\right]=0 .
\end{aligned}
$$

Compared to the Virasoro case, it may not be unexpected that the increased wealth of Jacobi identities will lessen the number of conditions below.

The simplest set of starting operators, as will be discussed at the end of the paper, consists of the operators $L_{-1}$ and $F_{1}$.

Lemma 9. $L_{-1}$ and $F_{1}$ reach all the $L_{n}$ 's and all the $F_{n}$ 's through the iterative definitions

$$
\begin{aligned}
& \text { (Def. a) } \quad F_{0}=-\frac{2}{3}\left[L_{-1}, F_{1}\right], \\
& \text { (Def. b) } \quad L_{2}=\frac{1}{2}\left\{F_{1}, F_{1}\right\}, \\
& \text { (Def. c) } \quad L_{1}=\frac{1}{3}\left[L_{2}, L_{-1}\right], \\
& \text { (Def. d) } \quad L_{0}=\frac{1}{2}\left[L_{1}, L_{-1}\right], \\
& \text { (Def.e) } \quad F_{-1}=-2\left[L_{-1}, F_{0}\right], \\
& \text { (Def. f) } \quad L_{-2}=\frac{1}{2}\left\{F_{-1}, F_{-1}\right\},
\end{aligned}
$$

supplemented by the recursive definitions (6) and (7) for $n>2$, together with the analogous ones

$$
\text { (Def) } F_{n+1}=\frac{2}{(2 n-1)}\left[F_{n}, L_{1}\right] \text { for } n>0 \text {, }
$$

for the positive indices larger than one, and

$$
\text { (Def) } F_{-n-1}=\frac{2}{(1-2 n)}\left[F_{-n}, L_{-1}\right] \text { for } n>0 \text {, }
$$

for the negative indices smaller than minus one. 
In order to simplify the proofs, we note the Ramond "parity" algebra automorphisms:

Lemma 10. The commutation relations (1) and (4) are invariant under the symmetry operation (10) complemented by

$$
F_{n} \rightarrow i F_{-n} .
$$

They are further invariant under a mere sign change of the fermion operators $F_{n}$, resulting from two applications of (30): $F_{n} \rightarrow-F_{n}$.

As in the Virasoro case,

Lemma 11. All the commutation relations with $L_{0}$ follow iteratively from the two conditions

$$
\begin{aligned}
\text { (Cond. a) } \quad\left[F_{1}, L_{0}\right] & =F_{1}, \\
\text { (Cond. b) }\left[L_{-1}, L_{0}\right] & =-L_{-1},
\end{aligned}
$$

and the definitions.

This again follows from repeated application of the graded version of Lemma 3 , i.e. use of $J 1(0, m, n)$ and $J 2(0, m, n)$.

In this case of the Ramond algebra, to evaluate all commutators involving the initial set of operators defined in (27), namely $L_{2}, L_{1}, L_{0}, L_{-1}, L_{-2}$ and $F_{1}, F_{0}, F_{-1}$ within itself, only one new condition has to be imposed. Indeed,

Lemma 12. With the condition

$$
\text { (Cond.c) }\left\{F_{0}, F_{0}\right\}=2 L_{0}-c \text {, }
$$

all the relevant commutation relations which remain within the starting set are fixed, and all the relevant Jacobi identities are satisfied.

Given the previous lemma, the proof of this one is direct, through a lengthy set of sequentially allowed Jacobi identities:

$$
\begin{aligned}
& j 1 \equiv J 2(-1,1,1) \\
& j 2 \equiv J 2(-1,1,0) \\
& j 3 \equiv J 2(-1,0,0) \\
& j 4 \equiv J 3(1,1,-1) \\
& j 5 \equiv J 3(1,-1,-1) \\
& j 6 \equiv J 3(0,0,1) \\
& j 7 \equiv J 2(2,-1,-1) \\
& j 8 \equiv J 3(0,1,-1) \\
& j 9 \equiv J_{2}(1,-1,-1) .
\end{aligned}
$$

The following Table (I) indicates which definitions, conditions, or Jacobi identities have been used in the obvious graded commutators entries. The dot entries below the diagonal are the symmetric to the ones above. $\times$ indicates that the entry is missing, as it falls outside the set in question:

Moreover, all the remaining commutation relations can be deductively determined, from merely two further conditions

$$
\begin{aligned}
\text { (Cond.d) }\left[L_{4}, F_{0}\right] & =2 F_{4}, \\
\text { (Cond.e) }\left[L_{-4}, F_{0}\right] & =-2 F_{-4},
\end{aligned}
$$

as there are more (graded) Jacobi identities which can be used. The proof follows 
Table I

\begin{tabular}{llllllll}
\hline & $L_{-2}$ & $L_{-1}$ & $L_{1}$ & $L_{2}$ & $F_{-1}$ & $F_{0}$ & $F_{1}$ \\
\hline \hline$L_{-2}$ & 0 & $\times$ & $j 9$ & $j 7$ & $\times$ & $\times$ & $j 5$ \\
\hline$L_{-1}$ & $\cdot$ & 0 & def.d & def. c & $\times$ & def. e & def. a \\
\hline$L_{1}$ & $\cdot$ & $\cdot$ & 0 & $\times$ & $j 8$ & $j 6$ & $\times$ \\
\hline$L_{2}$ & $\cdot$ & $\cdot$ & $\cdot$ & 0 & $j 4$ & $\times$ & $\times$ \\
\hline$F_{-1}$ & $\cdot$ & $\cdot$ & $\cdot$ & $\cdot$ & def.f & $j 3$ & $j 2$ \\
\hline$F_{0}$ & $\cdot$ & $\cdot$ & $\cdot$ & $\cdot$ & $\cdot$ & cond. c & $j 1$ \\
\hline$F_{1}$ & $\cdot$ & $\cdot$ & $\cdot$ & $\cdot$ & $\cdot$ & $\cdot$ & def. b \\
\hline
\end{tabular}

the same lines as in the Virasoro case. We first look at the positive level, starting with the commutators of the L's with the $F$ 's.

Lemma 13. All the commutators of fixed total level $k=m+n$, with $m>0, n \geqq 0$, between the positive L's and the non-negative F's can be obtained from the definitions (27-29) together with conditions $(31,32,34)$.

Proof. This is again proved by induction on $k$. Define as in (14) above the $r-2$ first $X_{t}^{(k)}, t=1, \ldots, r-2$ for $k=2 r$ even; and the $r-1$ first $X_{t}^{(k)}, t=1, \ldots, r-1$ for $k=2 r+1$ odd. Now complete this set by $r+1 X_{t}^{(k)}$ 's $, t=r-1, \ldots, 2 r-1$, for $k$ even:

$$
X_{t}^{(k=2 r)} \equiv\left\{F_{t-r+1}, F_{3 r-t-1}\right\} ;
$$

and by $r+1 X_{t}^{(k)}$ 's, $t=r, \ldots, 2 r$ for $k$ odd:

$$
X_{t}^{(k=2 r+1)} \equiv\left\{F_{t-r}, F_{3 r-t+1}\right\} .
$$

Moreover, define the $k-1$ commutators

$$
Y_{t}^{(k)} \equiv\left[L_{t+1}, F_{k-t-1}\right]
$$

for $t=1, \ldots, k-1$.

The $k-1$ commutators $Y^{(k)}$ satisfy the matrix equation

$$
N_{s t}^{(k)} Y_{t}^{(k)}=n_{s}^{(k)} F_{k},
$$

where the first $k-2$ rows of the matrix $N^{(k)}$ are obtained from the Jacobi identities $J 1(1, s+1, k-s-2)$ for $s=1, \ldots, k-2$, and the last row from $J 3(1,1, k-2)$ (these are allowed, as the commutators $X$ and $Y$ are known for lower levels by the 
induction):

$$
N^{(k)}=\left(\begin{array}{ccccccc}
7-2 k & -2 & 0 & \cdots & 0 & 0 & 0 \\
0 & 9-2 k & -4 & \cdots & 0 & 0 & 0 \\
0 & 0 & 11-2 k & \cdots & 0 & 0 & 0 \\
\vdots & \vdots & \vdots & \vdots & \vdots & \vdots & \vdots \\
0 & 0 & 0 & \cdots & -1 & 6-2 k & 0 \\
0 & 0 & 0 & \cdots & 0 & 1 & 4-2 k \\
1 & 0 & 0 & \cdots & 0 & 2 & 0
\end{array}\right)
$$

while the vector $n^{(k)}$ has components

$$
n_{s}^{(k)}=\left(k-\frac{3}{2} s-\frac{5}{2}\right)(2 k-3)
$$

for its first $k-2$ elements, and

$$
n_{k-1}^{(k)}=0
$$

for its last element. The determinant of the matrix is

$$
\operatorname{det} N^{(k)}=(k-2)\left(2^{k-2}(k-3) !-(-1)^{k} 2^{6-k} \frac{(2 k-7) !}{(k-4) !}\right)
$$

and is nonvanishing for $k>4$, hence Eq. (38) can be inverted to give the $Y^{(k)}$ 's in terms of $F_{k}$ in agreement with (4).

The $k=2,3$, and 4 cases are treated individually. For $k=2$, Lemma 12 ensures that $J 3(0,1,1)$ is allowed, and this fixes $\left[L_{2}, F_{0}\right]$ in terms of the definition of $F_{2}$. For $k=3, J 1(1,2,0)$ and $J 3(1,1,1)$ dictate the vanishing of $\left[L_{2}, F_{1}\right]$ and fix $\left[L_{3}, F_{0}\right]$ in terms of the definition of $F_{3}$. For $k=4, J 1(1,2,1)$ and $J 3(1,1,2)$ lead to the same identity, which, together with the only remaining allowed one, $J 1(1,3,0)$, amount to two relations for three unknowns: this is why (Cond.d) had to be imposed in (34).

For the $X^{(k)}$ unknowns, the even and odd cases are separated.

Lemma 14. All the $X^{(k)}$ 's, i.e. the commutators of two positive level L's and anticommutators of two positive level $F$ 's at level $k>0$ are obtainable by induction on $k$.

Proof. It is assumed in the induction that the $X$ 's are known for levels lower than $k$, while the $Y$ 's are known up to $k$ by the previous lemma. The $X^{(k)}$ 's satisfy a matrix equation:

$$
P_{s t}^{(k)} X_{t}^{(k)}=p_{s}^{(k)} L_{k}
$$

For even $k=2 r>4$, the matrix 


$$
\begin{aligned}
& P^{(k=2 r)=} \\
& \left(\begin{array}{cccccccccccc}
4-k & -1 & 0 & \cdots & 0 & 0 & 0 & 0 & 0 & \cdots & 0 & 0 \\
0 & 5-k & -2 & \cdots & 0 & 0 & 0 & 0 & 0 & \cdots & 0 & 0 \\
0 & 0 & 6-k & \cdots & 0 & 0 & 0 & 0 & 0 & \cdots & 0 & 0 \\
\vdots & \vdots & \vdots & \vdots & \vdots & \vdots & \vdots & \vdots & \vdots & \vdots & \vdots & \vdots \\
0 & 0 & 0 & \cdots & -r & 3-r & 0 & 0 & 0 & \cdots & 0 & 0 \\
0 & 0 & 0 & \cdots & 0 & 1-r & 0 & 0 & 0 & \cdots & 0 & 0 \\
0 & 0 & 0 & \cdots & 0 & 0 & 2 k-3 & -1 & 0 & \cdots & 0 & 0 \\
0 & 0 & 0 & \cdots & 0 & 0 & 0 & 2 k-5 & 1 & \cdots & 0 & 0 \\
0 & 0 & 0 & \cdots & 0 & 0 & 0 & 0 & 2 k-7 & \cdots & 0 & 0 \\
\vdots & \vdots & \vdots & \vdots & \vdots & \vdots & \vdots & \vdots & \vdots & \vdots & \vdots & \vdots \\
0 & 0 & 0 & \cdots & 0 & 0 & 0 & 0 & 0 & \cdots & k-1 & k-3 \\
2 & 0 & 0 & \cdots & 0 & 0 & 0 & 0 & 0 & \cdots & k-4 & 0
\end{array}\right)
\end{aligned}
$$

The first $r-2$ rows follow from the allowed $J(1, s+1, k-s-2)$, the next $r$ rows from $J 2(1, s-r+1,3 r-s-2)$, and the last row from $J 2(2, r-1, r-1)$. The vector $p_{s}^{(k=2 r)}$ has

$$
p_{s}^{(k=2 r)}=(2 s+3-k)(2-k)
$$

for its first $r-2$ components, $s=1, \ldots, r-2$;

$$
p_{s}^{(k=2 r)}=4(k-2)
$$

for its next $r$ components $s=r-1, \ldots, k-2$; and $p_{k-1}^{(k=2 r)}=0$ for its last one.

$$
\operatorname{det} P^{(k=2 r)}=(-)^{r+1} \frac{(k-4)}{2^{r}} \frac{(2 k-3) !}{(k-1) !}
$$

is nonvanishing, so that the unknowns $X^{(k=2 r)}$ are determined in terms of $L_{k}$.

The $k=2$ case is specified by (Def. b) and $J 2(1,1,0) . k=4$ requires a nontrivial bottom row, such as $(1,-3,0)$ which follows from $J 2(3,1,0)$, and yields a determinant of 14 .

For odd $k=2 r+1>7$, the $(k-1) \times(k-1)$ matrix $P^{(k=2 r+1)}$ is a blockreduction - a direct sum $\oplus$ - of a $(r-1) \times(r-1)$ matrix $P_{I}^{(k=2 r+1)}$, which turns out to be identical to $M^{(k=2 r+1)}$ of the Virasoro case $(19)$, and a $(r+1) \times(r+1)$ matrix $P_{I I}^{(k=2 r+1)}$. Specifically,

$$
\begin{aligned}
& P^{(k=2 r+1)}=\left(\begin{array}{ccccccccccccc}
k-4 & 1 & 0 & 0 & \cdots & 0 & 0 & 0 & 0 & 0 & \cdots & 0 & 0 \\
0 & k-5 & 2 & 0 & \cdots & 0 & 0 & 0 & 0 & 0 & \cdots & 0 & 0 \\
0 & 0 & k-6 & 3 & \cdots & 0 & 0 & 0 & 0 & 0 & \cdots & 0 & 0 \\
0 & 0 & 0 & k-7 & \cdots & 0 & 0 & 0 & 0 & 0 & \cdots & 0 & 0 \\
\vdots & \vdots & \vdots & \vdots & \vdots & \vdots & \vdots & \vdots & \vdots & \vdots & \vdots & \vdots & \vdots \\
0 & 0 & 0 & 0 & \cdots & r & r-2 & 0 & 0 & 0 & \cdots & 0 & 0 \\
8-k & k-7 & 0 & 1 & \cdots & 0 & 0 & 0 & 0 & 0 & \cdots & 0 & 0 \\
0 & 0 & 0 & 0 & \cdots & 0 & 0 & 2 k-3 & -1 & 0 & \cdots & 0 & 0 \\
0 & 0 & 0 & 0 & \cdots & 0 & 0 & 0 & 2 k-5 & 1 & \cdots & 0 & 0 \\
0 & 0 & 0 & 0 & \cdots & 0 & 0 & 0 & 0 & 2 k-7 & \cdots & 0 & 0 \\
\vdots & \vdots & \vdots & \vdots & \vdots & \vdots & \vdots & \vdots & \vdots & \vdots & \vdots & \vdots & \vdots \\
0 & 0 & 0 & 0 & \cdots & 0 & 0 & 0 & 0 & 0 & \cdots & k & k-4 \\
0 & 0 & 0 & 0 & \cdots & 0 & 0 & 0 & 0 & 0 & \cdots & 0 & 2 k-4
\end{array}\right) .
\end{aligned}
$$


The first $r-2$ rows follow from $J(1, s+1, k-s-2)$, the $r-1$ 'th row from $J(2,3, k-5)$ (for $k>7)$, and the last $r+1$ rows from $J 2(1, s-r+1,3 r-s-1)$. The vector $p^{(k=2 r+1)}$ has components

$$
p_{s}^{(k=2 r+1)}=(2 s+3-k)(2-k)
$$

for $s=1, \ldots, r-2 ; p_{r-1}^{(k=2 r+1)}=0$; and

$$
p_{s}^{(k=2 r+1)}=4(k-2)
$$

for the remaining $r+1$ components. The determinant,

$$
\operatorname{det} P^{(k=2 r+1)}=(-)^{r+1} \frac{k(k-7)}{3 \cdot 2^{r+1}} \frac{((r-2) !)^{2}(2 k-3) !}{(k-4) !(k-3) !}
$$

is again nonzero. The unique result (4) follows as usual. Note that for $k=9$ the third row is $(1,-2,1)$ instead, but the conclusion holds.

Cases $k=1,3,5$, and 7 are treated individually. $k=1$ is included in the starting set. $k=3$ has only two unknowns, fixed by $J 2(1,0,2)$ and $J 2(1,1,1)$. For $k=5$, there are four unknowns, specified by $J 2(1,0,4), J 2(1,1,3), J 2(1,2,2)$, and $J 2(2,0,3)$.

whilst

$$
P^{(5)}=\left(\begin{array}{cccc}
0 & 7 & -1 & 0 \\
0 & 0 & 5 & 1 \\
0 & 0 & 0 & 6 \\
2 & 2 & 0 & -1
\end{array}\right),
$$

$$
p^{(5)}=(12,12,12,0) \text {, }
$$

and the determinant is nonvanishing $(-420)$.

Finally, for $k=7, J(1,2,4), J 2(1, s, 6-s)$, for $s=0,1,2,3$, and $J 2(2,0,5)$ yield

$$
P^{(7)}=\left(\begin{array}{cccccc}
-6 & -2 & 0 & 0 & 0 & 0 \\
0 & 0 & 11 & -1 & 0 & 0 \\
0 & 0 & 0 & 9 & 1 & 0 \\
0 & 0 & 0 & 0 & 7 & 3 \\
0 & 0 & 0 & 0 & 0 & 10 \\
2 & 0 & 4 & 0 & -1 & 0
\end{array}\right)
$$

with nonzero determinant (27720), and

$$
p^{(7)}=(20,20,20,20,20,0) \text {. }
$$

Lemmas 13 and 14 extend to negative levels by the "parity" automorphism of Lemma 10 which holds for the assumptions underlying their proof.

Lemma 15. All graded commutators between positive and negative level operators also satisfy the Ramond algebra relations.

The proof includes that of Lemmas 7 and 8 and extends it to $\left\{F_{m}, F_{-m}\right\}$ and $\left\{F_{m}, F_{1-m}\right\}$ inductively through $J 2(1, m,-m)$ and $J 2(-1, m+1,-m)$, starting with $m=1 .\left\{F_{m}, F_{-n}\right\}$ for all positive $n<m$ follows inductively in $m$ from 
$J 2(1, m,-n)$ and the above, starting with $m=2$, and likewise for its parity image. $\left[F_{m}, L_{-n}\right]$ is also specified inductively in $m$ : if it is known for all positive $n$, for a given $m$, then it follows for $m+1$ through $J 1(1,-n, m)$, the induction basis being $m=0$, known by Lemma 13, whilst its parity image of Lemma 10 follows likewise.

The above lemmas are finally assembled to yield

Theorem 2. Given $L_{-1}$ and $F_{1}$ and the definitions of (27-29) together with the five conditions $(31,32,34)$, the Ramond algebra of (1) and (4) is satisfied.

\section{The Neveu-Schwarz Algebra}

We finally treat the Neveu-Schwarz algebra $(1,4)$ where the indices $a$ and $b$ run over the half-integers. Following convention, the fermionic generators are called $G$. The simplest set of starting generators consists of the operators $G_{3 / 2}$ and $L_{-2}$. Define the initial $L_{n}$ 's and $G_{a}$ 's through the iterative definitions

$$
\begin{array}{rlrl}
(\text { Def. } \alpha) & {\left[G_{3 / 2}, L_{-2}\right]} & =\frac{5}{2} G_{-1 / 2}, \\
(\text { Def. } \beta) & \left\{G_{-1 / 2}, G_{-1 / 2}\right\} & =2 L_{-1}, \\
& (\text { Def. } \gamma) & {\left[G_{3 / 2}, L_{-1}\right]} & =2 G_{1 / 2}, \\
& \text { (Def. } \delta \text { ) } & \left\{G_{1 / 2}, G_{1 / 2}\right\} & =2 L_{1}, \\
& \text { (Def. } \varepsilon) & {\left[G_{-1 / 2}, L_{1}\right]} & =-G_{1 / 2}, \\
\text { (Def. } \zeta) & \left\{G_{1 / 2}, G_{3 / 2}\right\} & =2 L_{2}, \\
\text { (Def. } \eta) & {\left[G_{1 / 2}, L_{-2}\right]} & =\frac{3}{2} G_{-3 / 2}, \\
\text { (Def. } \theta) & {\left[L_{1}, L_{-1}\right]} & =2 L_{0} .
\end{array}
$$

Further define the higher $L_{n}$ 's recursively through (6) and (7) for $n>1$;

$$
\text { (Def) } G_{a+1}=\frac{2}{(2 a-1)}\left[G_{a}, L_{1}\right] \text { for } a>1 / 2 \text {, }
$$

for the G's of positive half-integer index larger then $3 / 2$; and also

$$
\text { (Def) } G_{-a-1}=\frac{2}{(1-2 a)}\left[G_{-a}, L_{-1}\right] \text { for } a>1 / 2
$$

for the $G$ 's of negative half-integer indices smaller than $-3 / 2$. The Neveu-Schwarz algebra also has the automorphism of Lemma 10 with the integer $n$ replaced by the half-integer $a$.

Lemma 16. All the commutation relations with $L_{0}$ follow iteratively from the two conditions:

$$
(\text { Cond. } \alpha) \quad\left[L_{0}, G_{3 / 2}\right]=-\frac{3}{2} G_{3 / 2}, \quad(\text { Cond. } \beta) \quad\left[L_{0}, L_{-2}\right]=2 L_{-2} .
$$

The proof is again a repeated application of Lemma 3 through $J 1(0, m, a), J 2(0, a, b)$ and the definitions (56-58).

Lemma 17. In order to close the initial set of operators defined in (56), namely $L_{-2}, L_{-1}, L_{0}, L_{1}, L_{2}, G_{-3 / 2}, G_{-1 / 2}, G_{1 / 2}, G_{3 / 2}$, within itself, three conditions have 
to be imposed. With the conditions

$$
\begin{array}{rlrl}
(\text { Cond. } \gamma) & {\left[L_{2}, L_{-2}\right]} & =4 L_{0}+6 c, \\
& (\text { Cond. } \delta) & \left\{G_{3 / 2}, G_{-1 / 2}\right\} & =2 L_{1}, \\
& \text { (Cond. } \varepsilon) & \left\{G_{-3 / 2}, G_{-1 / 2}\right\} & =2 L_{-2},
\end{array}
$$

all the relevant commutation relations within this set are fixed, and all the relevant Jacobi identities are satisfied.

Given the previous lemma, the proof is direct, based on the following sequence of Jacobi identities:

$$
\begin{array}{rlrl}
i 1 & \equiv J 3(1 / 2,1 / 2,1 / 2) & i 2 & \equiv J 2(1,-1 / 2,-1 / 2) \\
i 3 & \equiv J 3(-1 / 2,-1 / 2,-1 / 2) & i 4 \equiv J 1(1,-1,-1 / 2) \\
i 5 & \equiv J 2(-2,3 / 2,1 / 2) & i 6 \equiv J 2(-1,3 / 2,1 / 2) \\
i 7 & \equiv J 3(3 / 2,-1 / 2,1 / 2) & i 8 & \equiv J 3(3 / 2,-1 / 2,-3 / 2) \\
i 9 & \equiv J 2(-2,1 / 2,1 / 2) & i 10 \equiv J 2(1,-1 / 2,-3 / 2) \\
i 11 & \equiv J 3(3 / 2,1 / 2,-3 / 2) . & &
\end{array}
$$

The following Table (II) summarizes the expressions specifying each graded commutator entry, in analogy to Table (I):

In order to close inductively the full algebra, four more conditions have to be imposed; for example,

$$
\begin{aligned}
& \text { (Cond. } \left.\zeta) \quad\left\{G_{3 / 2}, G_{3 / 2}\right\}=2 L_{3}, \quad \text { (Cond. } \eta\right) \quad\left\{G_{3 / 2}, G_{7 / 2}\right\}=2 L_{5} \text {, } \\
& \text { (Cond. } \left.\theta) \quad\left\{G_{-3 / 2}, G_{-3 / 2}\right\}=2 L_{-3} \text {, (Cond. } l\right) \quad\left\{G_{-3 / 2}, G_{-7 / 2}\right\}=2 L_{-5} \text {. }
\end{aligned}
$$

Lemma 18. All the commutators of fixed total level $d=q+1 / 2$, where $q$ is an integer, between the positive L's and the positive G's can be obtained from the definitions (56-58), together with the conditions $(59,60,62)$.

\section{Table II}

\begin{tabular}{lllllllll}
\hline & $L_{-2}$ & $L_{-1}$ & $L_{1}$ & $L_{2}$ & $G_{-3 / 2}$ & $G_{-1 / 2}$ & $G_{1 / 2}$ & $G_{3 / 2}$ \\
\hline$L_{-2}$ & 0 & $\times$ & $i 9,10$ & cond. $\gamma$ & $\times$ & $\times$ & def. $\eta$ & def. $\alpha$ \\
\hline$L_{-1}$ & $\cdot$ & 0 & def. $\theta$ & $i 6$ & $\times$ & $i 3$ & $i 4$ & def. $\gamma$ \\
\hline$L_{1}$ & $\cdot$ & $\cdot$ & 0 & $\times$ & $i 8$ & def. $\varepsilon$ & $i 1$ & $\times$ \\
\hline$L_{2}$ & $\cdot$ & $\cdot$ & $\cdot$ & 0 & $i 11$ & $i 7$ & $\times$ & $\times$ \\
\hline$G_{-3 / 2} \cdot$ & $\cdot$ & $\cdot$ & $\cdot$ & $\times$ & cond. $\varepsilon$ & $i 9,10$ & $i 5$ \\
\hline$G_{-1 / 2}$ & $\cdot$ & $\cdot$ & $\cdot$ & $\cdot$ & $\cdot$ & def. $\beta$ & $i 2$ & cond. $\delta$ \\
\hline$G_{1 / 2}$ & $\cdot$ & $\cdot$ & $\cdot$ & $\cdot$ & $\cdot$ &. & def. $\delta$ & def. $\zeta$ \\
\hline$G_{3 / 2}$ & $\cdot$ & $\cdot$ & $\cdot$ & $\cdot$ & $\cdot$ &. &. & $\times$ \\
\hline
\end{tabular}


Proof. Define the $q-1$ unknown commutators $Y^{(d)}$ between the positive L's and the positive $G$ 's of total level $d=q+1 / 2$ by

$$
Y_{s}^{(d=q+1 / 2)} \equiv\left[L_{s+1}, G_{d-s-1}\right]
$$

for $s=1, \ldots, q-1$. As before, for the $X$ 's separate the even from the odd cases. For $k=2 r$ even, let the $r-2$ first $X_{t}^{(k=2 r)}$ 's, $(t=1, \ldots, r-2)$, i.e. the commutators of two positive L's, be given by

$$
X_{t}^{(k=2 r)} \equiv\left[L_{t+1}, L_{k-t-1}\right],
$$

and the next $r$ unknowns $X_{t}^{(k=2 r)},(t=r-1, \ldots, 2 r-2)$, the anticommutators between two positive $G$ 's, by

$$
X_{t}^{(k=2 r)} \equiv\left\{G_{3 / 2+t-r}, G_{3 r-t-3 / 2}\right\} .
$$

For $k=2 r+1$ odd, let the $r-1$ first $X_{t}^{(k=2 r+1)}$, with $t=1, \ldots, r-1$, be given by

$$
X_{t}^{(k=2 r+1)} \equiv\left[L_{t+1}, L_{k-t-1}\right],
$$

and the next $r+1$ unknowns, for $t=r, \ldots, 2 r$, be given by

$$
X_{t}^{(k=2 r+1)} \equiv\left\{G_{1 / 2+t-r,}, G_{3 r-t+1 / 2}\right\} .
$$

Assume that the $X$ 's and the $Y$ 's are known for all positive levels smaller than $d=q+1 / 2$. It is then proved that all the $Y_{t}^{(d=q+1 / 2)}$ are determined.

For $q>5$, the $q-1$ unknowns $Y$ obey the matrix equation

$$
V_{s t}^{(d=q+1 / 2)} Y_{t}^{(d)}=v_{s}^{(d)} G_{d},
$$

where the $q-2$ first rows of the $(q-1) \times(q-1)$ matrix $V^{(d=q+1 / 2)}$ are given by the $q-2$ Jacobi identities $J 1(1,1+s, d-s-2)$ for $s=1, \ldots, q-2$, and the last row by the identity $J 3(3 / 2,3 / 2, d-3)$ :

$$
\left(\begin{array}{cccccccc}
3-q & -1 & 0 & 0 & \cdots & 0 & 0 & 0 \\
0 & 4-q & -2 & 0 & \cdots & 0 & 0 & 0 \\
0 & 0 & 5-q & -3 & \cdots & 0 & 0 & 0 \\
0 & 0 & 0 & 6-q & \cdots & 0 & 0 & 0 \\
\vdots & \vdots & \vdots & \vdots & \vdots & \vdots & \vdots & \vdots \\
0 & 0 & 0 & 0 & \cdots & -1 & 3-q & 0 \\
0 & 0 & 0 & 0 & \cdots & 0 & 0 & 2-q \\
0 & 2 & 0 & 0 & \cdots & 0 & 4 & 0
\end{array}\right)
$$

The first $q-2$ components of the vector $v^{(d)}$ are

$$
v_{s}^{(d=q+1 / 2)}=(q-2-3 s / 2)(q-1)
$$

for $s=1, \ldots, q-2$, and the last component is zero

$$
v_{q-1}^{(d=q+1 / 2)}=0
$$

Since the determinant of $V$ is nonvanishing,

$$
\operatorname{det} V^{(q+1 / 2)}=2\left(3-q-(-)^{q} 2\right)(q-2) !,
$$

the equation (68) can be inverted, yielding the desired result. 
The case of $q=1$ (i.e. $d=3 / 2$ ) is already contained in the initial set. For the case $q=2$ (i.e. $d=5 / 2$ ), the only unknown, $\left[L_{2}, G_{1 / 2}\right]$, is obtained from the definition of $G_{3 / 2}$ through the use of the Jacobi identity $J 3(1 / 2,1 / 2,3 / 2)$. For the case of $q=3$ (i.e. $d=7 / 2$ ), the two unknowns $\left[L_{2}, G_{3 / 2}\right]$ and $\left[L_{3}, G_{1 / 2}\right]$ are determined from the two Jacobi identities $J 1(1,2,1 / 2)$ and $J 3(1 / 2,3 / 2,3 / 2)$. For $q=4$ the last row is $(0,2,0)$, also linearly independent, and the determinant is 4 . For $q=5$ the last row is $(0,2,4,0)$, which is proportional to the second row, so $J 3(1 / 2,3 / 2,7 / 2)$ is used instead, producing an independent last row $(1,0,1,1)$, and the determinant is -12 .

The $X$ unknowns are obtained by similar arguments.

Lemma 19. All the commutators of a given total level $k$ between two positive L's and the anticommutators between positive G's follow from conditions (60).

Proof. Assume that the $X$ 's and the $Y$ 's for all positive levels below $k$ are known. The $X$ unknowns satisfy the matrix equation

$$
U_{s t}^{(k)} X_{t}^{(k)}=u_{s}^{(k)} L_{k}
$$

It is again convenient to separate the even and odd cases.

For the $k=2 r$ even case, $(k>4)$ the $(k-2) \times(k-2)$ matrix $U^{(k=2 r)}$ has its first $r-2$ rows determined by the Jacobi identities $J(1, s+1, k-s-2),(s=1, \ldots, r-2)$, and its next $r$ rows specified by $J 2(1,3 / 2+s-r, 3 r-s-5 / 2),(s=r-1, \ldots, 2 r-2)$. In some analogy to $P^{(k=2 r+1)}$, it is a block-reduction of a $(r-2) \times(r-2)$ matrix $U_{I}^{(k=2 r)}$ and a $r \times r$ matrix $U_{I I}^{(k=2 r)}$. Specifically,

$$
U_{I}^{(k=2 r)}=\left(\begin{array}{ccccccc}
k-4 & 1 & 0 & \cdots & 0 & 0 & 0 \\
0 & k-5 & 2 & \cdots & 0 & 0 & 0 \\
0 & 0 & k-6 & \cdots & 0 & 0 & 0 \\
\vdots & \vdots & \vdots & \vdots & \vdots & \vdots & \vdots \\
0 & 0 & 0 & \cdots & r+1 & r-4 & 0 \\
0 & 0 & 0 & \cdots & 0 & r & r-3 \\
0 & 0 & 0 & \cdots & 0 & 0 & r-1
\end{array}\right)=M^{(k=2 r)}
$$

and

$$
U_{I I}^{(k=2 r)}=\left(\begin{array}{cccccccc}
k-2 & 0 & 0 & 0 & \cdots & 0 & 0 & 0 \\
0 & k-3 & 1 & 0 & \cdots & 0 & 0 & 0 \\
0 & 0 & k-4 & 2 & \cdots & 0 & 0 & 0 \\
0 & 0 & 0 & k-5 & \cdots & 0 & 0 & 0 \\
\vdots & \vdots & \vdots & \vdots & \vdots & \vdots & \vdots & \vdots \\
0 & 0 & 0 & 0 & \cdots & r+1 & r-3 & 0 \\
0 & 0 & 0 & 0 & \cdots & 0 & r & r-2 \\
0 & 0 & 0 & 0 & \cdots & 0 & 0 & 2(r-1)
\end{array}\right) .
$$

The vector $u_{s}^{(k=2 r)}$ has as its first $r-2$ components

$$
u_{s}^{(k=2 r)}=(3+2 s-k)(k-2)
$$


for $s=1, \ldots, r-2$; and as its $r$ next components,

$$
u_{s}^{(k=2 r)}=2(k-2)
$$

for $s=r-1, \ldots, 2 r-2$. The determinant of $U$

$$
\operatorname{det} U^{(k=2 r)}=2 \frac{(k-4) !(k-2) !}{(r-2) !(r-2) !}
$$

is different from zero. Hence (73) can be inverted, producing the proper unique result.

The case $k=2$ is already contained in the initial set. The general case also extends trivially to the case $k=4$ : the matrix $U^{I}$ is simply absent and the two unknowns $\left\{G_{1 / 2}, G_{7 / 2}\right\}$ and $\left\{G_{3 / 2}, G_{5 / 2}\right\}$ are obtained through the $U^{I I}$ part of the matrix only.

The case $k=2 r+1$ odd is more complex. Indeed for $k>7$, using the $r-2$ allowed Jacobi identities $J(1, s+1, k-s-2)$ for $s=1, \ldots, r-2$ to fix the $r-2$ first rows of the matrix $U^{(k=2 r+1)}, J(2,3, k-5)$ to fix the $(r-1)$ 'th row, $J 2(1,1 / 2+s-r, k-s+r-3 / 2)$ for the rows $s=r, \ldots, 2 r-1$ and $J 2(k-3,3 / 2,3 / 2)$ for the last, $2 r^{\prime}$ th, line yields

$$
\begin{aligned}
& U^{(k=2 r+1)}=
\end{aligned}
$$

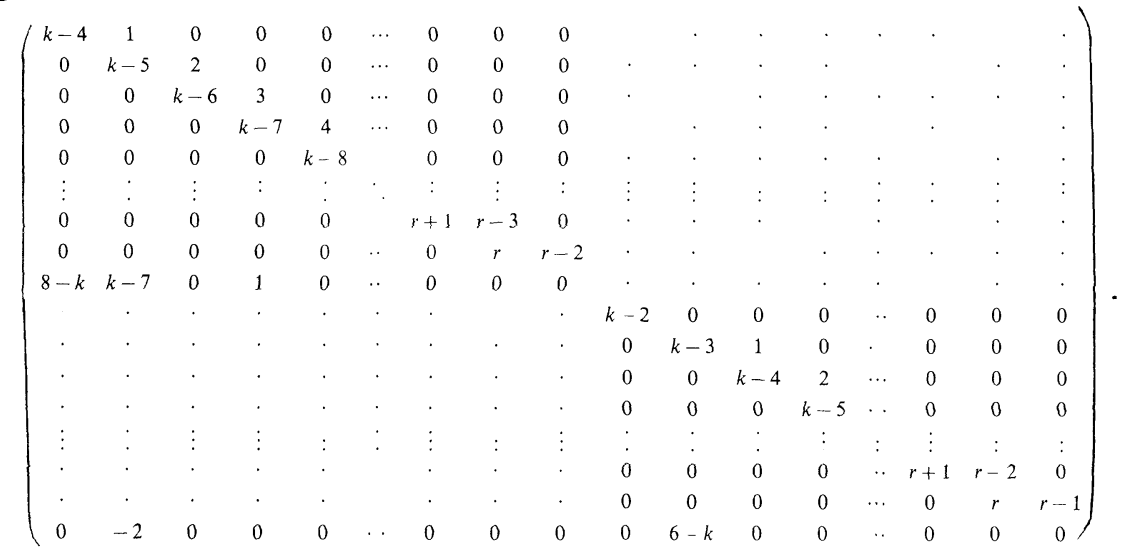

Note that the dotted blocks are zero matrices of dimension $(r-1) \times(r+1)$ in the upper-right corner, and $r \times(r-1)$ in the lower-left corner above the last row, respectively.

The vector $u^{(k=2 r+1)}$ has

$$
u_{s}^{(k=2 r+1)}=(3+2 s-k)(k-2)
$$

for its first $r-2$, i.e. $s=1, \ldots, r-2$, components; zero

$$
u_{r-1}^{(k=2 r+1)}=0
$$

for its $r-1$ 'th component;

$$
u_{s}^{(k=2 r+1)}=2(k-2)
$$

for its next $r$ components, i.e. $s=r, \ldots, 2 r-1$; and zero again for its last 
component

$$
u_{2 r}^{(k=2 r+1)}=0 .
$$

The determinant of the matrix $U^{(k=2 r+1)}$

$$
\operatorname{det} U^{(k=2 r+1)}=k(k-2)^{2}(6-k)(k-7)(r-1) !(r-2) ! / 3 !
$$

is nonvanishing and hence the usual result follows.

The cases of $k=1,3,5,7$ are treated individually. $k=1$ is in the starting set. For $k=3$, there are two unknowns, $X_{1}^{(3)}=\left\{G_{1 / 2}, G_{5 / 2}\right\}$ and $X_{2}^{(3)}=\left\{G_{3 / 2}, G_{3 / 2}\right\}$. The two allowed Jacobi identities $J 2(1,1 / 2,3 / 2)$ and $J 2(2,1 / 2,1 / 2)$ lead to the same relation, fixing only $X_{1}^{(3)}$. Hence (Cond. $\zeta$ ) and its parity image (Cond. $\theta$ ) are introduced.

For $k=5$, for the four unknowns $X_{1}^{(5)}=\left[L_{2}, L_{3}\right], \quad X_{2}^{(5)}=\left\{G_{1 / 2}, G_{9 / 2}\right\}$, $X_{3}^{(5)}=\left\{G_{3 / 2}, G_{7 / 2}\right\}$, and $X_{4}^{(5)}=\left\{G_{5 / 2}, G_{5 / 2}\right\}$, the six allowed Jacobi identities, namely $J 2(1,1 / 2,7 / 2), J 2(1,3 / 2,5 / 2), J 2(2,1 / 2,5 / 2), J 2(2,3 / 2,3 / 2), J 2(3,1 / 2,3 / 2)$, and $J 2(4,1 / 2,1 / 2)$, give only three independent relations, as can be checked directly. Hence, (Cond. $\eta$ ) and its parity image (Cond. $\iota$ ) are required.

Finally, the case $k=7$ can be solved without further constraint. The six unknowns obey the matrix equation (73), where a $6 \times 6$ matrix $U^{(7)}$ is, for example, provided by the following six Jacobi identities: $J(1,2,4), J 2(1,1 / 2,11 / 2)$, $J 2(2,1 / 2,9 / 2), J 2(2,3 / 2,7 / 2), J 2(2,5 / 2,5 / 2)$, and $J 2(5,1 / 2,3 / 2)$.

$$
U^{(7)}=\left(\begin{array}{cccccc}
-3 & -1 & 0 & 0 & 0 & 0 \\
0 & 0 & 5 & 0 & 0 & 0 \\
4 & 0 & 7 & 0 & -1 & 0 \\
4 & 0 & 0 & 5 & 0 & 1 \\
2 & 0 & 0 & 0 & 3 & 0 \\
-2 & 0 & -1 & -2 & 0 & 0
\end{array}\right)
$$

with

$$
\operatorname{det} U^{(7)}=-140
$$

and the vector $u^{(7)}$ has

$$
u_{s}^{(7)}=10
$$

for its first two components $s=1,2$, and zero for its remaining four components for $s=3,4,5,6$,

$$
u_{s}^{(7)}=0
$$

Extension to the negative level parity images proceeds in the standard manner.

Lemma 20. All graded commutators between positive and negative level operators also satisfy the Neveu-Schwarz algebra.

The proof includes that of Lemmas 7 and 8, and parallels that of Lemma 15: $\left\{G_{a}, G_{-a}\right\}$ and $\left\{G_{a}, G_{1-a}\right\}$ follow inductively from $J 2(1, a,-a)$ and $J 2(-1, a+1,-a)$, starting with $a=3 / 2$; then, $\left\{G_{a}, G_{-b}\right\}$ for all positive $b \leqq a$ 
follows inductively in $a$ from $J 2(1, a,-b)$ and the above, starting with $a=3 / 2$. $\left[G_{1 / 2}, L_{-n}\right]$ for all positive $n$ follows from $J 1(1,-n,-1 / 2)$ and the parity image of Lemma 18; $\left[G_{3 / 2}, L_{-n}\right]$ is determined inductively in $n$ through $J 1(-1,-n, 3 / 2)$, starting with $n=2$. Hence, starting with $a=3 / 2$, induction in $a$ determines $\left[G_{a}, L_{-n}\right]$ for all positive $n$ through $J 1(1,-n, a)$; likewise for its parity image.

All the preceding lemmas are collected in the following theorem:

Theorem 3. Given the starting generators $G_{3 / 2}$ and $L_{-2}$ and the definitions (56-58) and the nine conditions $(59,60,62)$, the entire Neveu-Schwarz algebra $(1,4)$ is satisfied.

\section{Discussion and Conclusions}

It has been demonstrated so far that the Virasoro, Ramond, and NeveuSchwarz algebras are built out of a mere subset of two generators, subject to a finite number of conditions: both the definitions of the remaining generators and the conditions imposed consist of nested (graded) commutators of these two starting generators.

It is evident however that, within the initial anadromic operator sets in the proof of the above theorems, there is an ambiguity concerning which are the starting operators, which are the definitions, which are the imposed conditions, and which are the derived relations. This suggests some freedom to reassign the status of selected relations in this set. For instance, in the Virasoro case, we could have started alternatively from three instead of two generators, namely $L_{2}, L_{-2}$, and $L_{-1}$. The definitions are then

$$
\begin{aligned}
& \text { (Def. 1') } L_{1}=\frac{1}{3}\left[L_{2}, L_{-1}\right], \\
& \text { (Def. 2') } L_{0}=\frac{1}{2}\left[L_{1}, L_{-1}\right],
\end{aligned}
$$

together with (6) for $n>1$ and (7). There are then nine conditions:

$$
\begin{array}{lr}
(\text { Cond. 1') } & {\left[L_{2}, L_{0}\right]=2 L_{2}} \\
(\text { Cond. 2') } & {\left[L_{-2}, L_{0}\right]=-2 L_{-2}} \\
(\text { Cond. 3') } & {\left[L_{-1}, L_{0}\right]=-L_{-1},} \\
\text { (Cond. 4') } & {\left[L_{2}, L_{-2}\right]=4 L_{0}+6 c} \\
\text { (Cond. 5') } & {\left[L_{-2}, L_{1}\right]=-3 L_{-1}}
\end{array}
$$

and the four conditions of (13). Both the number of starting generators and the number of conditions have increased by one. The bulk of the inductive proof of Theorem 1 remains unchanged.

In a somewhat different vein, also in the Virasoro case, note that Condition 4 could be turned into a definition of $c$, now regarded as an abstract operator. One way of writing this would then be to replace Condition 4 by an alternate definition and two alternate conditions:

$$
\begin{aligned}
& \text { (Alt. Def. 5) } \quad c=\frac{1}{6}\left(\left[L_{2}, L_{-2}\right]-2\left[L_{1}, L_{-1}\right]\right) \\
& \text { (Alt. Cond. 4) } \quad\left[c, L_{3}\right]=0 \\
& \text { (Alt. Cond. } \left.4^{\prime}\right)\left[c, L_{-2}\right]=0 .
\end{aligned}
$$


With this alternate set, $c$ belongs to the center of the enveloping algebra and hence can be diagonalized. This way of defining $c$ as a new abstract operator rather than as a multiple of the identity increases the number of conditions by one.

Exploring the general properties of such reassignments is beyond the scope of our present paper. In such a study, it should be interesting to search for invariants of reassignments, as extra starting generators may obviate definitions but, as in the above example, increase the number of conditions. A topological or graphtheoretical meaning to the numbers of conditions found above is not excluded. At this point, however, we have not shown in complete generality that no other choice of starting generators and conditions could reduce the size of the above sets. What we will explore below is the adequacy of starting sets of generators for defining the infinite algebras.

Consider the bosonic Virasoro algebra first. The general requirement for the starting set is, of course, that it contain both negative and positive indices, and that taking sums of the starting indices yield all integers, excluding sums corresponding to trivial commutators. Naturally, the starting set should be minimal, in the sense that no subset of these operators should suffice by itself to define all operators of the algebra. Thus, e.g. if the starting set is to consist of only two generators, a positive index $m$ is required, together with a negative one $-n$. However, neither can have an absolute value of 1 , since this truncates the positive, or negative, subalgebra at that level. Moreover, if $m$ and $n$ have a common divisor $r$, then only multiples of $r$ may be reached through the sums specified. We are thus led to:

Proposition 1. $L_{m}$ and $L_{-n}$ are an adequate starting set for relatively prime $m, n>1$.

This is shown for $m>n$, whilst the parity image statement follows likewise. Since $L_{1}$ and $L_{-1}$ ladder to all indices from $m$ and $-n$, it suffices to reach these two operators. Positive indices may be lowered by multiples of $n$, so that we consider modulo $n$ the $n$ integers $0 \cdot m, 1 \cdot m, \ldots,(n-1) m$. No two integers in this set can be equal, since otherwise $p m=q m \bmod n$, so that $(p-q) m=s n$ with $p-q<n$, which would dictate that $m$ and $n$ share a common factor. Thus, in this set of $n$ different numbers, 1 and $n-1$ (leading to -1 ) occur.

When the assumptions of the above proposition are not satisfied, more starting generators are required to produce initial operators of the required type and form a minimal starting set. In general, the largest common divisor of all starting generators should clearly not be larger than one, as in the example (89--90).

For two starting generators, the Ramond algebra affords two options. Firstly, the starting set may contain an $L_{-m}$ and an $F_{n}$, or the corresponding parity image choice, with $m, n>0$. In this case, $m$ must be odd, otherwise no odd-indexed bosonic generators would be reachable. Since $L_{2 n}$ is reachable, the problem reduces to the previous one for $m$ and $n$ relatively prime, albeit $m=1$ is now acceptable; for $m$ and $n$ sharing a common divisor, all derived fermions will be indexed by multiples of that divisor. The second option involves $F_{-m}$ and $F_{n}$. In this case, out of $m, n>0$, one must be even and the other odd, otherwise no odd-indexed bosonic operators would be reachable. Furthermore, $m$ and $n$ should be relatively prime, otherwise only multiples of their common factor would index all bosonic generators. 
For two starting generators, the Neveu-Schwarz algebra also affords two options. For a starting set $L_{-n}$ and $G_{m+1 / 2}$ with $m, n>0$, it is also necessary that $n>1$, as $L_{-1}$ cannot be lowered by $G_{-1 / 2}$; moreover, $n$ and $2 m+1$ must be relatively prime, as usual. For a starting set of $G_{-(m+1 / 2)}$ and $G_{n+1 / 2}, m, n>0$, as above, and it is likewise seen that $m$ and $n$ must be relatively prime. When there are more than two starting generators, the options are more numerous, but the generalization is straightforward.

Our construction, by dint of carrying the entire content of the algebra (1) - or (1) and (4) - implicitly solves an analog of the word problem [5] for these infinite algebras, namely the algorithmic determination of all "words," in our case sequences of nested commutators of the starting generators (or these generators themselves), and the specification of equivalence of words. Similar constructions may apply to Kac-Moody algebras. The significance of the formulation at hand lies in the fact that every relation involving, e.g., the Virasoro algebra and its consequences may alternatively be written in terms of the two starting generators subject to the above 8 conditions, and thus any use of the algebra (1) may now be supplanted by Jacobi identities. Thus several constructions based on the Virasoro algebra may simplify, and new ones may well emerge. At present, however, we have not utilized this to explore the representation structure of the algebra, which should be the subject of a separate investigation. For example, it should be interesting to search for ways in which $c$ may be constrained, e.g. as it is known to be for special representations. Given our construction, a natural question which arises includes relaxing the form of the conditions posited above, in various ways, thereby inducing extensions of the Virasoro and super-Virasoro algebras, like those of Ref. [6], or potentially consistent new infinite algebras.

Acknowledgements. We record our appreciation to E Corrigan, Y Meurice, and A. Kumar for stimulating questions.

\section{References}

1. Virasoro, M.: Phys. Rev. D1, 2933 (1970); Fubini, S., Veneziano, G.: Ann. Phys. 63, 12 (1971); I Gel'fand, D. Fuks: Funkts. Anal. Prilozhen. 2, 92 (1968); Kirillov, A : Funkts. Anal Prilozh. 15, 75 (1981); Segal, G.: Commun. Math. Phys 80, 301 (1981); Friedan, D, Qiu, Z, Shenker, S : Commun. Math. Phys. 107, 535 (1986); Beilinson, A, Manin, Yu., Schechtman, V.: Sheaves of the Virasoro and Neveu-Schwarz Algebras. In: Springer Lecture Notes in Mathematics: K-Theory, Airthmetic, and Geometry, vol. 1289, Manin, Yu. (ed.) Berlin, Heidelberg, New York: Springer 1987; Witten, E.: Commun. Math. Phys. 114, 1 (1988); Fairlie, D., Nuyts, J, Zachos, C.: Phys. Lett. B202, 320 (1988)

2. For a review, see: Goddard, P., Olive, D : Int. J Mod. Phys. A1, 303 (1986)

3. Ramond, P.: Phys. Rev. D3, 2415 (1971); Neveu, A., Schwarz, J.: Nucl. Phys. B31, 86 (1971)

4. Meurice, Y.: Nucl. Phys. B276, 325 (1986)

5. Magnus, W., Karass, A., Solitar, D.: Combinatorial group theory: presentations of groups in terms of generators and relations. New York: Dover 1976; Boone, W.: Ann. Math. 70, 207 (1959); Novikov, P.: Am. Math. Soc. Trans. (2) 9, 1 (1958) (original in Trudy Mat. Inst. im. Steklov 44, Izdat. Akad. Nauk. SSSR, 143 (1955))

6. Krichever, I., Novikov, S.: Funct. Anal. Appl. 21, 126 (1987) (original in Funkts Anal Prilozh., p. 46); Floratos, E., Iliopoulos, J : Phys Lett B201, 237 (1988)

Communicated by L. Alvarez-Gaumé

Received February 8, 1988 